\title{
A phase II trial of concurrent 3D-CRT/IMRT and oxaliplatin, 5-fluorouracil and leucovorin (FOLFOX) in gastric cancer patients with R0 gastrectomy and D2 lymph node dissection
}

\author{
Xin Wang $\cdot$ Yali Shen $\cdot$ Hong Zhu $\cdot$ Yaqin Zhao $\cdot$ Zhiping Li $\cdot$ Meng Qiu $\cdot$ \\ Qiu Li · Hongfeng Gou • Yu Yang • Dan Cao · Jiyan Liu • Cheng Yi • \\ Zhengyin Liao $\cdot$ Deyun Luo $\cdot$ Feng Bi $\cdot$ Feng Xu
}

Received: 3 September 2014/Accepted: 29 December 2014/Published online: 22 January 2015

(C) The International Gastric Cancer Association and The Japanese Gastric Cancer Association 2015

\begin{abstract}
Background To evaluate the safety and efficacy of a concurrent three-dimensional conformal radiotherapy (3DCRT) or intensity-modulated radiotherapy (IMRT) plus oxaliplatin, 5-fluorouracil and leucovorin (FOLFOX) regimen in completely resected gastric cancer patients with D2 lymph node dissection.

Materials and methods Patients with stage IB-IIIC gastric cancer (per the AJCC, 7th edition) who had undergone R0 and D2 gastrectomy were recruited. Two cycles of FOLFOX with concurrent 3D-CRT or IMRT (50.4 Gy/28f) were administered. One and an additional five cycles of FOLFOX were delivered before and after concurrent chemoradiotherapy, respectively. Primary endpoints were relapse-free survival (RFS) and overall survival (OS), with adverse events as secondary endpoints.

Results From 2008 to 2011, 110 patients were evaluable. The 1-, 2- and 3-year RFS and OS were 86.2, 72.2, 67.8 and $94.7,87.2,77.6 \%$, respectively. On multivariate analysis, stage ( $\leq$ IIIA vs. $>$ IIIA) was a statistically significant factor affecting both RFS and OS. Additionally, the $\mathrm{T}$-category $(\leq \mathrm{T} 4 \mathrm{a}$ vs. $=\mathrm{T} 4 \mathrm{~b})$ was a statistically
\end{abstract}

X. Wang · Y. Shen $\cdot$ H. Zhu $\cdot$ Y. Zhao $\cdot$ Z. Li · M. Qiu .

Q. Li · H. Gou - Y. Yang - D. Cao - J. Liu - C. Yi - Z. Liao ·

D. Luo · F. Bi · F. Xu ( $\square)$

Department of Abdominal Oncology, Cancer Center, West

China Hospital, Sichuan University, No. 37 of Wainan Guoxue

Lane, Wuhou District, Chengdu 610041, Sichuan Province,

People's Republic of China

e-mail:18980601780@163.com

X. Wang $\cdot$ Y. Shen

State Key Laboratory of Biotherapy, Cancer Center, West China Hospital, Sichuan University, Chengdu, Sichuan Province, China significant factor affecting only the RFS. The most commonly observed grade 3 or 4 adverse events were nausea and vomiting, decreased appetite, leukopenia/neutropenia and fatigue, each of which occurred in 14.5, 11.8, 9.1 and $6.4 \%$ patients, respectively.

Conclusions Adjuvant 3D-CRT/IMRT to a dose of $50.4 \mathrm{~Gy} / 28 \mathrm{f}$ with concurrent FOLFOX is safe and effective in patients following radical gastrectomy with D2 lymph node dissection.

Keywords Gastric cancer - D2 lymph node dissection · Adjuvant chemoradiotherapy $\cdot$ 3D-CRT $\cdot$ IMRT

\section{Introduction}

Gastric cancer is the fourth most common cancer and the third leading cause of cancer-related death worldwide [13]. Although surgery provides the best chance of cure, locoregional and distant recurrence rates after a curativeintent operation are still high $[4,5]$. To improve the outcomes of patients with resectable gastric cancer, several treatment strategies, such as adjuvant chemotherapy, adjuvant chemoradiotherapy (CRT) and perioperative chemotherapy, have been incorporated into clinical decision-making following the publication of studies demonstrating improved survival [5-8]. Adjuvant CRT is the standard of care for resectable gastric cancer patients in the USA based on the outcome of the US Intergroup 0116 (INT-0116) trial [5, 9]. In this study, the overall survival (OS) (HR 1.32, $p=0.0046)$ and relapse-free survival (RFS) (HR 1.51, $p<0.001$ ) were reported to benefit significantly from adjuvant CRT with 10 years of follow-up [9]. However, only $10 \%$ of the patients in this study received a D2 lymph node dissection. In centers where D2 
dissection is performed routinely, the incorporation of adjuvant CRT remains controversial.

The INT-0116 trial used a conventional opposed AP/PA technique. More sophisticated radiotherapy techniques, such as intensity-modulated radiotherapy (IMRT) and three-dimensional conformal radiotherapy (3D-CRT), have been used more frequently in recent years and have been reported to be dosimetrically superior to conventional radiotherapy $[10,11]$.

The INT-0116 used a chemotherapy regimen of 5-fluorouracil and leucovorin (5-FU/FA). Oxaliplatin has been demonstrated to have a synergistic effect with radiotherapy for digestive tract cancers in both preclinical and clinical studies [12-14]. As is well known, the FOLFOX regimen, which contains oxaliplatin, leucovorin and 5-fluorouracil, is the standard treatment for colon cancer. A prospective randomized phase II trial reported that adjuvant FOLFOX could result in significantly better 3-year OS than 5-FU/FA (30.0 vs. 16.0 months, $p<0.05$ ) in gastric cancer patients after curative-intent gastrectomy [15]. Further studies have demonstrated that the FOLFOX regimen is an effective and well-tolerated treatment for patients with gastric cancer [16].

These findings provide a strong rationale to test the adjuvant combination of 3D-CRT/IMRT and FOLFOX for gastric cancer patients who have undergone D2 lymph node dissection. We previously reported our phase I results demonstrating that adjuvant 3D-CRT (50.4 Gy) with concurrent FOLFOX (oxaliplatin: $65 \mathrm{mg} / \mathrm{m}^{2}$ ) for gastric cancer was feasible and safe [17]. Here we report the results of a prospective phase II trial evaluating the safety and efficacy of adjuvant 3D-CRT/IMRT and concurrent FOLFOX for completely resected gastric cancer patients with D2 lymph node dissection.

\section{Materials and methods}

\section{Enrollment and eligibility}

Patients were enrolled in this phase II study based on the following eligibility criteria: age between 18 and 75 years; Eastern Cooperative Oncology Group performance status of 0 or 1 ; histologically confirmed adenocarcinoma of the stomach; had undergone a complete gastrectomy without residual disease (R0 gastrectomy) with D2 lymph node dissection of no more than 3 months prior to starting treatment on protocol; stage IB-IIIC per the 7th edition of the AJCC staging manual; adequate bone marrow/liver/ kidney function. Patients with known metastatic disease, a history of other malignancies or an inability to tolerate treatment because of other systemic illnesses were excluded from the study. This study protocol was approved by the West China Hospital institutional review board, and all patients provided written informed consent before study enrollment. The trial was registered at the Chinese Clinical Trial Registry (ChiCTR-ONC-12001918).

\section{Surgery}

In order to be eligible for the study, all patients were required to have undergone a complete resection with negative margins as well as a D2 lymph node dissection with at least 15 lymph nodes removed. No routine splenectomy or pancreatic tail resection was performed. The procedure entailed resection of either the involved proximal or distal part of the stomach or the entire stomach, as necessary, as well as the perigastric lymph nodes and celiac, hepatic arterial, splenic or splenic-hilar, and cardial lymph nodes, depending on the location of the primary tumor.

\section{Radiotherapy}

All patients were treated in the supine position. CT simulation was performed for every patient using a helical CT scan with 3-mm slices and intravenous contrast. The clinical target volume (CTV) for radiotherapy was defined as the tumor bed, remnant stomach, all anastomoses and stumps, and the draining lymph nodes. The design of the CTV for each patient was individualized depending on the primary tumor, involved lymph nodes and the type of surgery performed. The planning target volume (PTV) incorporated a $1.0-\mathrm{cm}$ margin around the CTV. The 3DCRT or IMRT techniques were used in all patients. The radiotherapy technique was determined by the treating physician based on the complexity of the target volume and the organs at risk (OAR). Treatment plans were generated using the Pinnacle treatment planning system (version 9.0, Philips Medical Systems, Madison, WI) using 6-MV X-rays and were delivered with an Elekta linear accelerator (Elekta Oncology Systems, Crawley, UK). The 3D-CRT plans were generated using 4-5 coplanar beams with beam angles dependent on the tumor location. Inverse step-and-shoot IMRT plans were generated using seven coplanar beams (whose angles were $0^{\circ}$, $52^{\circ}, 104^{\circ}, 156^{\circ}, 204^{\circ}, 256^{\circ}$ and $308^{\circ}$, respectively) with an average of 40 segments. The prescribed dose was 50.4 Gy to the PTV, administered in 28 daily fractions of $1.8 \mathrm{~Gy}, 5$ days per week. Plans were acceptable if the prescribed dose covered $>95 \%$ of the PTV and no more than $1 \mathrm{cc}$ received $>107 \%$ of the prescribed dose. Dose constraints of critical organs were as follows: liver $\mathrm{V} 30<30 \%$ [18]; two-thirds of one kidney less than $18 \mathrm{~Gy}$ or $30 \%$ of each kidney volume of each kidney less than $25 \mathrm{~Gy}$; spinal cord Dmax $<40$ Gy. Image-guided 
radiotherapy (IGRT) and active breathing control (ABC) were allowed in order to decrease the geometric uncertainty and target motion.

\section{Chemotherapy}

Beginning 4-12 weeks after surgery, eligible patients received one cycle of adjuvant FOLFOX4 (oxaliplatin $85 \mathrm{mg} / \mathrm{m}^{2}$, day 1 ; CF $200 \mathrm{mg} / \mathrm{m}^{2}, 2 \mathrm{~h}$, day $1-2 ; 5-\mathrm{Fu}$ $400 \mathrm{mg} / \mathrm{m}^{2}$, i.v. bolus; 5 -Fu $600 \mathrm{mg} / \mathrm{m}^{2}$, continuous i.v. $22 \mathrm{~h}$, day 1-2) or mFOLFOX6 (oxaliplatin $85 \mathrm{mg} / \mathrm{m}^{2}$, day 1 ; CF $200 \mathrm{mg} / \mathrm{m}^{2}, 2 \mathrm{~h}$, day $1 ; 5-\mathrm{Fu} 400 \mathrm{mg} / \mathrm{m}^{2}$, i.v. bolus, d1; $5-\mathrm{Fu} 2,400 \mathrm{mg} / \mathrm{m}^{2}$, continuous i.v. $46 \mathrm{~h}$ ) before starting radiotherapy. Concurrent chemoradiotherapy began 14 days after the start of the initial cycle of chemotherapy, with two cycles of FOLFOX delivered on day 1 and day 22 of radiotherapy, respectively. The dosage of oxaliplatin delivered concurrently with radiotherapy was $65 \mathrm{mg} / \mathrm{m}^{2}$, per our phase I study [17]. After concurrent chemoradiotherapy, five additional cycles of full-dose FOLFOX chemotherapy were administered at 2-week intervals, starting 3-4 weeks after the completion of radiotherapy.

\section{Assessment of toxicity and follow-up}

Toxicity was assessed weekly during treatment using the National Cancer Institute Common Terminology Criteria for Adverse Events, version 3.0 [19], except for peripheral neuropathy where we used Levi's specific scale instead [20]. After adjuvant treatment, patients were followed up with surveillance physical examinations, laboratory tests and computed tomography scans every 3 months for the first 2 years, then every 6 months for the next 3 years.

\section{Dose modification}

If grade $3 / 4$ hematologic toxicities occurred, administration of chemotherapy was delayed until hematologic toxicities recovered to grade $0-1$, and the dose of 5-Fu was reduced by $25 \%$. If grade $\geq 2$ nonhematologic toxicities occurred, the administration of chemotherapy was also delayed until the toxicities decreased to grade $0-1$. During concurrent chemoradiotherapy, administration of chemotherapy was discontinued if grade 4 toxicities relating to chemotherapy occurred, except alopecia, and radiotherapy was continued when toxicities recovered to grade $0-1$. Radiotherapy was delayed if grade 3 radiation dermatitis occurred until it recovered to grade $0-1$. If patients required a delay of more than 3 weeks for recovery, treatment was discontinued, and the patients were treated outside the study.
Statistical analysis

The primary endpoints of this study were RFS and OS, with adverse events as secondary endpoints. The 3-year RFS rate was predicted to be improved from 50 to $65 \%$, with the assumption of a 3-year dropout rate of $15 \%$. The hypotheses were: $H 0: \mathrm{p}_{0}=0.50$ versus $H 1: \mathrm{p}_{1}=0.65$, where $p$ equals the 3-year RFS rate. A sample size of 107 patients was planned to record 62 RFS events with onesided $\alpha=0.05$ and $90 \%$ power. RFS was defined as the time between surgery and death or the first evidence of disease recurrence. OS was defined as the time between surgery and death. Patients who did not experience recurrence or die were censored at the date of last follow-up. The Kaplan-Meier method was used to estimate RFS and OS rates. Patient characteristics associated with RFS and OS were identified using univariate Cox regression analysis for RFS and OS. Significant variables in the univariate analysis were used in the multivariate analysis for RFS and OS to take the correlations among them into account. Demographic, baseline and toxicity data were summarized using descriptive statistics. The total doses of 5-Fu and oxaliplatin administered divided by the total planned dose were used as dose intensity. For all analyses, a $p$ value of $<0.05$ was considered statistically significant. All tests of statistical significance were two-sided. Statistical analysis was conducted using PASW Statistics (SPSS, IBM Corp.).

\section{Results}

Patient characteristics and treatment delivery

Between October 2008 to June 2011, 115 patients were recruited to the study. Three patients withdrew consent because of refusing adjuvant treatment; two patients were deemed ineligible because they were found to have abdominal lymph node metastases during baseline CT imaging prior to treatment. The remaining 110 patients were included in the analysis. Patient characteristics are summarized in Table 1. All patients received curative resection for gastric cancer with D2 lymph node dissection. The number of retrieved lymph nodes ranged from 15 to 103 (Table 1). Concurrent chemoradiotherapy was completed as planned by $90 \%$ of patients (99 of 110). The overall course of treatment was completed as planned by $72.7 \%$ of patients ( 80 of 110 ). A total of 30 patients did not complete the overall course of treatment for the following reasons: patient refusal of further radiotherapy (2 patients) or chemotherapy during treatment (8 patients), patient refusal because of toxicities (18 patients) and other causes including loss to follow-up ( 2 patients). The actual dose intensity was $84 \%$ of the planned doses for 5 -Fu and 
Table 1 Patient characteristics

\begin{tabular}{|c|c|}
\hline Characteristic & Value \\
\hline \multicolumn{2}{|l|}{ Age (years) } \\
\hline Median (range) & $55(23-73)$ \\
\hline \multicolumn{2}{|l|}{$\operatorname{Sex}(\%)$} \\
\hline Male & $82(74.5 \%)$ \\
\hline Female & $28(25.5 \%)$ \\
\hline \multicolumn{2}{|l|}{ Tumor site $(\%)$} \\
\hline Proximal & $37(33.7 \%)$ \\
\hline Body & $32(29.1 \%)$ \\
\hline Antrum & $30(27.3 \%)$ \\
\hline Multiple/diffuse & $11(10.0 \%)$ \\
\hline \multicolumn{2}{|l|}{ Maximum tumor diameter $(\mathrm{cm})$} \\
\hline Median & 4.8 \\
\hline Range & $0.7-12$ \\
\hline \multicolumn{2}{|l|}{ Number of retrieved lymph nodes } \\
\hline Median & 30 \\
\hline Range & $15-103$ \\
\hline \multicolumn{2}{|c|}{ Primary tumor classification stage $(\%)^{\mathrm{a}}$} \\
\hline IB & $6(5.5 \%)$ \\
\hline IIA & $6(5.5 \%)$ \\
\hline IIB & $10(9.1 \%)$ \\
\hline IIIA & $12(10.9 \%)$ \\
\hline IIIB & $25(22.7 \%)$ \\
\hline IIIC & $51(46.4 \%)$ \\
\hline \multicolumn{2}{|l|}{ T-category $(\%)^{\mathrm{a}}$} \\
\hline $\mathrm{T} 1 \mathrm{~b}$ & $4(3.6 \%)$ \\
\hline $\mathrm{T} 2$ & $13(11.8 \%)$ \\
\hline $\mathrm{T} 3$ & $9(8.2 \%)$ \\
\hline $\mathrm{T} 4 \mathrm{a}$ & $64(58.2 \%)$ \\
\hline $\mathrm{T} 4 \mathrm{~b}$ & $20(18.2 \%)$ \\
\hline \multicolumn{2}{|l|}{$\mathrm{N}$-category $(\%)^{\mathrm{a}}$} \\
\hline No & $9(8.2 \%)$ \\
\hline N1 & $19(17.3 \%)$ \\
\hline $\mathrm{N} 2$ & $35(31.8 \%)$ \\
\hline $\mathrm{N} 3 \mathrm{a}$ & $28(25.5 \%)$ \\
\hline $\mathrm{N} 3 \mathrm{~b}$ & $19(17.3 \%)$ \\
\hline \multicolumn{2}{|l|}{ Histology (\%) } \\
\hline Tubulous & $27(24.5 \%)$ \\
\hline Signet-ring cell & $45(40.9 \%)$ \\
\hline $\operatorname{Mix}$ & $38(34.5 \%)$ \\
\hline \multicolumn{2}{|l|}{ Tumor grade $(\%)$} \\
\hline G1: well differentiated & $0(0 \%)$ \\
\hline G2: moderately differentiated & $18(16.4 \%)$ \\
\hline G3: poorly differentiated & $92(83.6 \%)$ \\
\hline \multicolumn{2}{|l|}{ Radiation technique } \\
\hline 3D-CRT & $68(61.8 \%)$ \\
\hline IMRT & $42(38.2 \%)$ \\
\hline \multicolumn{2}{|l|}{ Chemotherapy regimen } \\
\hline FOLFOX4 & $67(60.8 \%)$ \\
\hline
\end{tabular}

Table 1 continued

\begin{tabular}{lc}
\hline Characteristic & Value \\
\hline mFOLFOX6 & $43(39.1 \%)$ \\
\hline $\begin{array}{l}\text { a American Joint Committee on Cancer Staging System, } 7 \text { th edition } \\
(2010)\end{array}$
\end{tabular}

$87 \%$ for oxaliplatin. IGRT and $\mathrm{ABC}$ were used for 26 patients. The follow-up rate was up to $93.6 \%$, and $6.4 \%$ of patients were lost to follow-up. The median follow-up time was 43 months (33-71 months).

\section{Relapse-free survival}

At the time of analysis, 35 patients $(31.8 \%)$ had experienced recurrence. Among them, two patients $(1.8 \%)$ with $\mathrm{N} 3 \mathrm{~b}$ disease developed locoregional and in-field recurrences. The remainder (33 patients, $30 \%$ ) developed distant metastases, which included distant lymph nodes, the peritoneum, liver and lung. Two patients developed another primary cancer (small cell lung cancer and renal clear cell carcinoma, respectively) after being treated for their gastric cancer. The 1-, 2- and 3-year RFS was 86.2, 72.2 and $67.8 \%$, respectively (Fig. 1a). The results of univariate analysis are shown in Table 2, where the T-category $(\leq \mathrm{T} 4 \mathrm{a}$ vs. $=\mathrm{T} 4 \mathrm{~b}), \mathrm{N}$-category $(\leq \mathrm{N} 2$ vs. $=\mathrm{N} 3)$, stage $(\leq$ IIIA vs. $>$ IIIA $)$ and tumor diameter $(\leq 4 \mathrm{~cm}$ vs. $>4 \mathrm{~cm}$ ) were significant factors for RFS. Other factors, including age, sex, tumor site, radiation technique, histology and tumor grade, did not demonstrate any statistically significant effect on RFS (Table 2). On multivariate analysis, only the $\mathrm{T}$-category $(\leq \mathrm{T} 4 \mathrm{a}$ vs. $=\mathrm{T} 4 \mathrm{~b})$ and stage $(\leq$ IIIA vs. $>$ IIIA) remained statistically significant factors affecting RFS (Table 3). Patients with $\mathrm{T} \leq \mathrm{T} 4 \mathrm{a}$ $(p=0.029)$ and stage $\leq$ IIIA $(p=0.03)$ had a significantly more favorable RFS versus those that did not. In a subgroup analysis, there were statistically significant differences in RFS among patients with different stages, and patients with an earlier stage had a more favorable RFS $(p=0.007$; Fig. 2a).

\section{Overall survival}

At the time of analysis, 27 patients $(24.5 \%)$ had died. The 1-, 2- and 3-year OS rates were 94.7, 87.2 and $77.6 \%$, respectively (Fig. 1b). Univariate analysis showed that the $\mathrm{T}$-category $(\leq \mathrm{T} 4 \mathrm{a}$ vs. $=\mathrm{T} 4 \mathrm{~b}), \mathrm{N}$-category $(\leq \mathrm{N} 2$ vs. $=\mathrm{N} 3)$, stage ( $\leq$ IIIA vs. $>$ IIIA) and age ( $<50$ vs. $\geq 50$ years) were significant factors for OS (Table 2). Other factors, including, sex, tumor site, tumor diameter, histology, radiation 

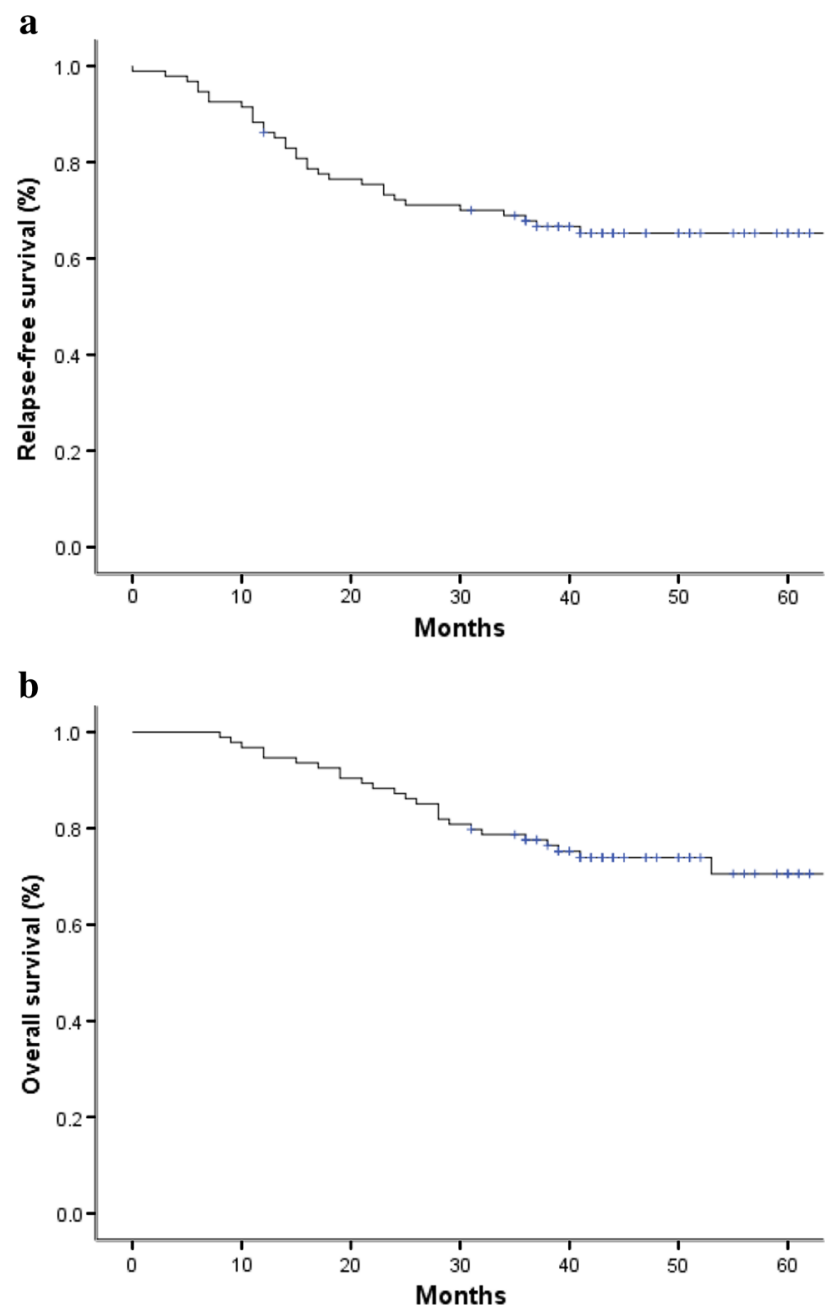

Fig. 1 Relapse-free survival (a) and overall survival (b) curves treated with adjuvant concurrent 3D-CRT/IMRT and FOLFOX

technique and tumor grade, did not demonstrate statistically significant effects (Table 2). Multivariate analysis showed that only stage ( $\leq$ IIIA vs. $>$ IIIA) remained a statistically significant factor affecting OS (Table 3). Patients with stage $\leq$ IIIA ( $p=0.029$ ) had a more favorable OS. In a subgroup analysis, there were statistically significant differences in OS among patients with different stages, and patients who had earlier stages had a more favorable OS $(p=0.018$; Fig. 2b).

Tolerability and safety

Table 4 shows acute grade 3 or 4 adverse events. The most commonly reported grade 3 or 4 adverse events in this phase II study were nausea and vomiting, decreased appetite, leukopenia/neutropenia and fatigue, each of which occurred in $14.5 \%, 11.8,9.1$ and $6.4 \%$ patients, respectively (Table 4). Grade 3 gastric hemorrhage, intestinal obstruction and allergic reaction to oxaliplatin each occurred in one $(0.9 \%)$ patient, respectively. Gastric hemorrhage occurred in one patient $(0.9 \%) 3$ months after the completion of concurrent chemoradiotherapy. Hemostasis was achieved by means of endoscopic intervention, and the patient recovered completely following blood transfusion. The patient with intestinal obstruction recovered completely with conservative management. No treatment-related death was recorded during the study. A total of $11.8 \%$ of patients (13 of 110 ) required a dose reduction of chemotherapy because of adverse events. However, none of them were serious adverse events (SAEs). Adverse events that led to patient refusal of further treatment occurred in $16.4 \%$ of patients (18 of 110). The most frequent adverse event causing treatment discontinuation was nausea and vomiting (10 patients). The other adverse events causing discontinuation were leucopenia/neutropenia (5 patients), anemia (1 patient), abdominal pain and stomatitis (1 patient), and an allergic reaction to oxaliplatin (1 patient). In general, these adverse events cause patients to discontinue the last 3-4 cycles of chemotherapy. No acute or late nephrotoxicity was observed.

\section{Discussion}

To date, although the role of adjuvant therapy has been clearly established in the management of resectable gastric cancer, the efficacy of adjuvant CRT after D2 lymph node dissection is still controversial. The use of advanced radiation techniques and more optimal chemotherapeutic agents in these patients needs to be evaluated. We conducted this prospective phase II trial to examine the safety and efficacy of adjuvant concurrent 3D-CRT/IMRT and FOLFOX in completely resected gastric cancer patients who had undergone D2 lymph node dissection based on our phase I dose-escalation study [17]. Our results suggest that this concurrent chemoradiotherapeutic regimen is acceptably safe and efficacious.

As discussed previously, INT-0116 clearly showed a survival advantage to the use of postoperative CRT. The 3-year RFS and OS in the CRT group were 48 and $50 \%$, compared with 31 and $41 \%$ in the surgery-only group ( $p<0.001$ and $p=0.005$, respectively) [5]. Several prospective trials have been published in recent years evaluating the benefit of adjuvant CRT in patients with D2 lymph node dissections [21-23]. A Korean phase III study applied the INT-0116 protocol to stage III-IV (M0) gastric cancer patients with D2 lymph node dissection [21]. However, this study showed that adjuvant CRT only improved locoregional recurrence-free survival (LRRFS) but not disease-free survival (DFS) or OS. The 5-year LRRFS, DFS and OS in the CRT group versus the chemotherapy-only group using intention-to-treat (ITT) 
Table 2 Univariate analysis: 3 -year RFS and OS according to subgroup

\begin{tabular}{|c|c|c|c|c|}
\hline \multirow[t]{2}{*}{ Factors } & \multicolumn{2}{|l|}{ RFS } & \multicolumn{2}{|l|}{ OS } \\
\hline & 3-year RFS (\%) & $p$ value & 3 -year OS (\%) & $p$ value \\
\hline Age (years) & & 0.353 & & 0.048 \\
\hline$<50$ & 73.3 & & 86.7 & \\
\hline$\geq 50$ & 62.3 & & 69.0 & \\
\hline Sex & & 0.593 & & 0.977 \\
\hline Male & 67.6 & & 73.1 & \\
\hline Female & 58.7 & & 66.0 & \\
\hline Tumor site & & 0.328 & & 0.299 \\
\hline Proximal & 74.5 & & 82.1 & \\
\hline Other sites & 61.7 & & 75.8 & \\
\hline Tumor diameter & & 0.036 & & 0.120 \\
\hline$\leq 4 \mathrm{~cm}$ & 73.4 & & 78.9 & \\
\hline$>4 \mathrm{~cm}$ & 51.5 & & 57.1 & \\
\hline Stage (combined) & & 0.000 & & 0.001 \\
\hline$\leq \mathrm{IIIA}$ & 93.4 & & 96.8 & \\
\hline$>$ IIIA & 55.1 & & 68.2 & \\
\hline T-category & & 0.001 & & 0.046 \\
\hline$\leq \mathrm{T} 4 \mathrm{a}$ & 73.2 & & 77.8 & \\
\hline$=\mathrm{T} 4 \mathrm{~b}$ & 29.3 & & 53.3 & \\
\hline $\mathrm{N}$-category & & 0.045 & & 0.018 \\
\hline$\leq \mathrm{N} 2$ & 74.1 & & 82.8 & \\
\hline$=\mathrm{N} 3$ & 57.4 & & 69.4 & \\
\hline Histology & & 0.977 & & 0.937 \\
\hline Tubulous & 83.3 & & 87.5 & \\
\hline Signet-ring cell & 54.4 & & 75.0 & \\
\hline Mix & 64.6 & & 76.1 & \\
\hline Tumor grade & & 0.313 & & 0.644 \\
\hline G2 & 76.6 & & 83.3 & \\
\hline G3 & 65.8 & & 76.3 & \\
\hline Radiation technique & & 0.602 & & 0.751 \\
\hline 3D-CRT & 65.5 & & 71.7 & \\
\hline IMRT & 71.7 & & 80.6 & \\
\hline Chemotherapy regimen & & 0.866 & & 0.699 \\
\hline FOLFOX4 & 69.9 & & 78.2 & \\
\hline mFOLFOX6 & 66.7 & & 70.7 & \\
\hline
\end{tabular}

analysis were 84.4 versus $62.7 \%(p=0.035), 60.9$ versus $50 \%(p=0.246)$ and 65.2 versus $54.6 \%(p=0.670)$, respectively [21]. This particular study used a conventional AP/PA radiotherapy technique. A prospective Chinese phase III study employed the same CRT scheme in gastric cancer patients with D2 dissection, but used IMRT instead [22]. In this study, it was reported that CRT improved RFS but not OS when compared with the chemotherapy-only group. The 5-year RFS and OS were 45.2 versus $35.8 \%$ $(p=0.029)$ and 48.4 versus $41.8 \%(p=0.122)$, respectively. The chemotherapy regimen used in all three of these trials was 5-FU/FA. We used the FOLFOX regimen in this phase II study to further explore the safety and efficacy of combined chemotherapy with radiotherapy. When compared with the results in the CRT groups of the three trials listed above, our results are comparable. In the ARTIST trial (a Korean phase III trial), where patients were treated with a conventional AP/PA technique plus capecitabine and cisplatin (XP), it was reported that the 3-year DFS in the CRT and chemotherapy-only groups was 78.2 versus $74.2 \%$, respectively $(p=0.0862)$ [23]. However, in a subgroup analysis of patients with positive lymph nodes, there was a statistically significant improvement in DFS in the CRT group (77.5 vs. 72.3 , respectively, $p=0.0365$ ). A 
Table 3 Multivariate analysis: RFS and OS according to subgroup

\begin{tabular}{|c|c|c|c|c|c|c|c|c|}
\hline \multirow[t]{3}{*}{ Factors } & \multicolumn{4}{|l|}{ RFS } & \multicolumn{4}{|l|}{ OS } \\
\hline & \multirow[t]{2}{*}{$p$ value } & \multirow[t]{2}{*}{$\operatorname{Exp}(B)$} & \multicolumn{2}{|c|}{$95 \%$ CI for $\operatorname{Exp}(B)$} & \multirow[t]{2}{*}{$p$ value } & \multirow[t]{2}{*}{$\operatorname{Exp}(B)$} & \multicolumn{2}{|c|}{$95 \%$ CI for $\operatorname{Exp}(B)$} \\
\hline & & & Lower & Upper & & & Lower & Upper \\
\hline \multicolumn{9}{|l|}{ Age } \\
\hline$<50$ versus $\geq 50$ years & 0.563 & 0.787 & 0.349 & 1.774 & 0.097 & 0.400 & 0.136 & 1.180 \\
\hline \multicolumn{9}{|l|}{ Tumor diameter } \\
\hline$\leq 4$ versus $>4 \mathrm{~cm}$ & 0.435 & 0.746 & 0.357 & 1.557 & 0.638 & 0.821 & 0.360 & 1.870 \\
\hline \multicolumn{9}{|l|}{ Stage } \\
\hline$\leq$ IIIA versus $>$ IIIA & 0.030 & 0.230 & 0.061 & 0.869 & 0.029 & 0.171 & 0.035 & 0.838 \\
\hline \multicolumn{9}{|l|}{ T-category } \\
\hline$\leq \mathrm{T} 4 \mathrm{a}$ versus $=\mathrm{T} 4 \mathrm{~b}$ & 0.029 & 0.418 & 0.191 & 0.915 & 0.177 & 0.536 & 0.217 & 1.324 \\
\hline \multicolumn{9}{|l|}{ N-category } \\
\hline$\leq \mathrm{N} 2$ versus $=\mathrm{N} 3$ & 0.922 & 0.963 & 0.454 & 2.043 & 0.747 & 0.869 & 0.372 & 1.324 \\
\hline
\end{tabular}

a

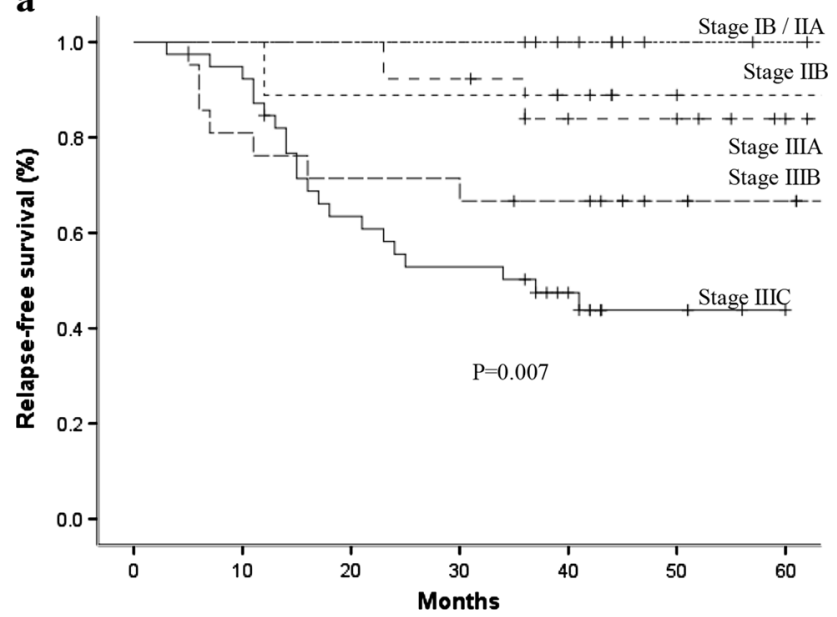

b

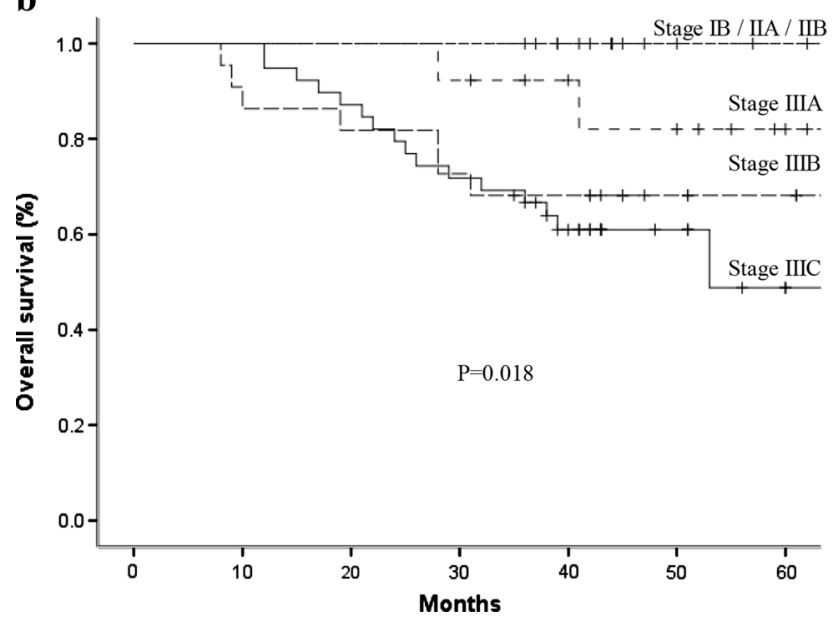

Fig. 2 Relapse-free survival (a) and overall survival (b) curves in subgroup analysis according to stage
Table 4 Adverse events profile (all grades)

\begin{tabular}{|c|c|c|c|c|}
\hline \multirow[t]{2}{*}{ Adverse events } & \multicolumn{2}{|c|}{ Grade $1-2$} & \multicolumn{2}{|c|}{ Grade $3-4$} \\
\hline & $N$ & $\%$ & $N$ & $\%$ \\
\hline Leucopenia/neutropenia & 38 & 34.5 & 10 & 9.1 \\
\hline Anemia & 46 & 41.8 & 1 & 0.9 \\
\hline Thrombocytopenia & 14 & 12.7 & 0 & 0 \\
\hline Nausea and vomiting & 63 & 57.3 & 16 & 14.5 \\
\hline Decreased appetite & 58 & 52.7 & 13 & 11.8 \\
\hline Diarrhea & 12 & 10.9 & 1 & 0.9 \\
\hline Fatigue & 32 & 29.1 & 7 & 6.4 \\
\hline Stomatitis & 23 & 20.9 & 5 & 4.5 \\
\hline Abdominal pain & 12 & 10.9 & 3 & 2.7 \\
\hline Peripheral sensory neuropathy & 9 & 8.2 & 0 & 0 \\
\hline ALT/AST increased & 15 & 13.6 & 0 & 0 \\
\hline Allergic reaction & 0 & 0 & 1 & 0.9 \\
\hline Gastric hemorrhage & 0 & 0 & 1 & 0.9 \\
\hline Intestinal obstruction & 0 & 0 & 1 & 0.9 \\
\hline
\end{tabular}

$A L T$ alanine aminotransferase, $A S T$ aspartate aminotransferase

further study evaluating the benefit of adjuvant concurrent CRT in patients with D2 lymph node dissection and positive lymph nodes is planned [23].

Recently, 3D-CRT or IMRT have been used more frequently in patients with gastric cancer. The feasibility and superiority of these techniques to conventional methods have likewise also been reported [10, 24-27]. There were several studies done that compared 3D-CRT with conventional techniques [25-27]. It was shown that 3D-CRT produces superior dose distributions and reduces the dose to organs at risk (OARs), with the potential to reduce treatment toxicity [25]. The radiation dose used in most of 
these studies was $45 \mathrm{~Gy} / 25 \mathrm{f}$. Higher doses, such as $50.4 \mathrm{~Gy} / 28 \mathrm{f}$, were also reported in the literature $[10,28]$, as well as our study. Milano et al. [10] used a dose of 50.4 Gy and compared IMRT, three-field 3D-CRT and a conventional AP/PA technique. They reported that IMRT could reduce the mean and above-threshold doses to OARs and was well tolerated [10]. Minn et al. [28] compared IMRT with 3D-CRT, with a median radiation dose of $45 \mathrm{~Gy}$ (range 41.4-54 Gy). It was reported that both of these techniques were well tolerated, and no significant differences were found in the 2-year OS (62 vs. $51 \%$, respectively, $\quad p=0.5)$ and grade $\geq 2$ acute gastrointestinal toxicity (61.5 vs. $61.2 \%$, respectively) between the two techniques. In our study, the results of univariate analysis revealed that the radiotherapy technique was not a significant factor for either RFS or OS.

Since these radiation techniques were shown to be both feasible and superior to conventional radiotherapy in patients with gastric cancer, several studies were performed to evaluate the outcomes of adjuvant IMRT/3D-CRT with concurrent chemotherapy utilizing either 5-FU or capecitabine [28-32]. Quero et al. [29] evaluated adjuvant concurrent 3D-CRT with 5-FU/FA and reported that the 5-year OS and DFS were 50 and $48 \%$, respectively. Leong et al. [30] used adjuvant 3D-CRT with concurrent 5-FU preceded and followed by adjuvant epirubicin, cisplatin and 5 -FU (ECF) and reported a 3-year OS of $61.6 \%$. In this study, $67 \%$ of patients completed the entire course of ECF, and $94 \%$ completed concurrent CRT as planned. Boda-Heggemann et al. [31] used adjuvant concurrent IMRT/3D-CRT with 5-FU/FA or capecitabine plus oxaliplatin (XELOX). The 5-year OS was reported to be 26 and $47 \%$ in the 3D-CRT and IMRT groups, respectively $(p=0.0693)$. Within the IMRT group, there was no significant difference in OS when comparing 5-FU/FA with XELOX. When compared with these retrospective studies, our results of RFS and OS in the single CRT arm (3DCRT/IMRT and FOLFOX) of this study are also comparable.

Many studies had found that lymph node involvement including the lymph node ratio (LNR) and N-category were associated with OS [33, 34] and RFS [33-36] in multivariate analyses. Considering all these results, as well as those from the ARTIST trial, the N-category was considered to be a very important prognostic factor. Additionally, the overall TNM stage group was also found to relate to the RFS [35] and OS in certain studies [22, 33, 35]. In our study, we found that the overall stage ( $\leq$ IIIA vs. $>$ IIIA) was a statistically significant factor affecting both RFS and OS; however, the T-category ( $\leq \mathrm{T} 4 \mathrm{a}$ vs. =T4b) was a statistically significant factor affecting only RFS, and the $\mathrm{N}$-category was not found to be significant. A possible reason for these results is that there were more patients with T4b disease and fewer patients with N0 and N1 disease in our study than in the other studies discussed. However, all of these predictive factors may be helpful for identifying patients who are at high risk.

The most common grade 3 or 4 adverse events reported in our study were nausea and vomiting (14.5\%), decreased appetite $(11.8 \%)$ and leukopenia/neutropenia $(9.1 \%)$. This is substantially fewer than the acute grade 3 or 4 adverse events, such as hematologic (54\%) and gastrointestinal $(33 \%)$ ones, that were reported in the CRT group of INT-0116 [5]. Conversely, the Chinese phase III trial of adjuvant 5-FU/FA plus IMRT vs. 5-FU/FA alone reported that grade 3-4 nausea and vomiting occurred in 2.7 and $1.6 \%$ of patients in CRT group, respectively, which was less than what was observed in our study [22]. The hematologic adverse events were similar to ours. A possible reason for the differences in reported adverse events may be the toxicity of multidrug chemotherapy. Leong et al. [30] delivered adjuvant concurrent 3D-CRT plus another combined chemotherapy (ECF) in a prospective study and reported grade 3/4 neutropenia (66\%) and gastrointestinal $(28 \%)$ toxicity was greater than what was observed in our study. In the ARTIST trial, grade 3 and 4 nausea in the CRT and chemotherapy-only groups were 12.3 and $12.4 \%$, respectively, which were similar to our results [23]. However, more grade 3 and 4 neutropenia was observed in the ARTIST study (48.4 and 40.7\%, respectively), which was also the most frequent adverse event requiring treatment modification. Eighteen patients (16.4\%) discontinued the treatment because of toxicities in our study, which was similar to the rate of $17 \%$ reported for the CRT group in the INT-0116 trial [5]. The whole treatment course was completed as planned by $72.7 \%$ of patients (80 of 110) in our study, and the concurrent chemoradiotherapy component was completed as planned by $90 \%$ of patients (99 of 110). This was greater than the completion rate of only $64 \%$ that was reported for the CRT group of the INT-0116 trial [5] and was similar to the rate of $75 \%$ observed in the CRT group of the ARTIST trial [23], suggesting that adjuvant 3D-CRT/IMRT to a dose of 50.4 Gy with concurrent FOLFOX can be safely administered to gastric cancer patients with D2 lymph node dissection. In addition, ten patients $(9.1 \%)$ discontinued the therapy because of refusal of further treatment in our study, which was also similar to the rate of $8 \%$ observed in the CRT group in the INT-0116 trial [5].

In subgroup analysis of our study, there were statistically significant differences in RFS and OS among patients with different stages, and patients who had an earlier stage resulted in more favorable RFS and OS $(p=0.007$ and 0.018 , respectively). Six patients had stage IB disease in this study; four of them were T1bN1M0 and two T2N0M0. These patients had the best RFS and OS according to the 
subgroup analysis. However, the enrollment of these patients, especially the patients with T2N0M0 disease, might reduce the value of this study because these patients generally have a good prognosis even without adjuvant treatment. Therefore, these patients should be excluded from future study.

The limitations of this study should also be addressed. The present study was a single-arm and single-institution study, which may increase the difficulty of generalizing the results. Most of the patients in this study refused using ABC and IGRT, and 4D CT was not available at our institution at that time, both of which could have contributed to greater geometric uncertainty for the radiotherapy. Additional work is needed to determine the proper PTV margin for these patients.

In conclusion, adjuvant 3D-CRT/IMRT to a dose of $50.4 \mathrm{~Gy} / 28 \mathrm{f}$ with concurrent FOLFOX is safe and effective in patients who have undergone radical gastrectomy with D2 lymph node dissection. A prospective randomized trial assessing the effects of adjuvant concurrent chemoradiotherapy in gastric cancer patients with high risk factors after D2 lymph node dissection is needed.

Acknowledgments This study was supported in part by the National Basic Research Program of China (project no. 2011CB935800).

Conflict of interest The authors report no conflict of interest.

\section{References}

1. Crew KD, Neugut AI. Epidemiology of gastric cancer. World J Gastroenterol. 2006;12:354-62.

2. Forman D, Burley VJ. Gastric cancer: global pattern of the disease and an overview of environmental risk factors. Best Pract Res Clin Gastroenterol. 2006;20:633-49.

3. Jemal A, Bray F, Center MM, Ferlay J, Ward E, Forman D. Global cancer statistics. CA Cancer J Clin. 2011;61:69-90.

4. Kim S, Lim DH, Lee J, Kang WK, MacDonald JS, Park CH, et al. An observational study suggesting clinical benefit for adjuvant postoperative chemoradiation in a population of over 500 cases after gastric resection with D2 nodal dissection for adenocarcinoma of the stomach. Int J Radiat Oncol Biol Phys. 2005;63:1279-85.

5. Macdonald JS, Smalley SR, Benedetti J, Hundahl SA, Estes NC, Stemmermann GN, et al. Chemoradiotherapy after surgery compared with surgery alone for adenocarcinoma of the stomach or gastroesophageal junction. N Engl J Med. 2001;345:725-30.

6. Cunningham D, Allum WH, Stenning SP, Thompson JN, Van de Velde CJ, Nicolson M, et al. Perioperative chemotherapy versus surgery alone for resectable gastroesophageal cancer. N Engl J Med. 2006;355:11-20.

7. Paoletti X, Oba K, Burzykowski T, Michiels S, Ohashi Y, Pignon JP, et al. Benefit of adjuvant chemotherapy for resectable gastric cancer: a meta-analysis. JAMA. 2010;303:1729-37.

8. Sakuramoto S, Sasako M, Yamaguchi T, Kinoshita T, Fujii M, Nashimoto A, et al. Adjuvant chemotherapy for gastric cancer with S-1, an oral fluoropyrimidine. $\mathrm{N}$ Engl $\mathrm{J}$ Med. 2007;357:1810-20.
9. Smalley SR, Benedetti JK, Haller DG, Hundahl SA, Estes NC, Ajani JA, et al. Updated analysis of SWOG-directed intergroup study 0116: a phase III trial of adjuvant radiochemotherapy versus observation after curative gastric cancer resection. J Clin Oncol. 2012;30:2327-33.

10. Milano MT, Garofalo MC, Chmura SJ, Farrey K, Rash C, Heimann $\mathrm{R}$, et al. Intensity-modulated radiation therapy in the treatment of gastric cancer: early clinical outcome and dosimetric comparison with conventional techniques. $\mathrm{Br} \mathrm{J}$ Radiol. 2006;79:497-503.

11. Ringash J, Perkins G, Brierley J, Lockwood G, Islam M, Catton $\mathrm{P}$, et al. IMRT for adjuvant radiation in gastric cancer: a preferred plan? Int J Radiat Oncol Biol Phys. 2005;63:732-8.

12. Cividalli A, Ceciarelli F, Livdi E, Altavista P, Cruciani G, Marchetti P, et al. Radiosensitization by oxaliplatin in a mouse adenocarcinoma: influence of treatment schedule. Int $\mathrm{J}$ Radiat Oncol Biol Phys. 2002;52:1092-8.

13. Khushalani NI, Leichman CG, Proulx G, Nava H, Bodnar L, Klippenstein D, et al. Oxaliplatin in combination with protractedinfusion fluorouracil and radiation: report of a clinical trial for patients with esophageal cancer. J Clin Oncology. 2002;20:2844-50.

14. Ajani JA. Evolving chemotherapy for advanced gastric cancer. Oncologist. 2005;10:49-58.

15. Zhang XL, Shi HJ, Cui SZ, Tang YQ, Ba MC. Prospective, randomized trial comparing 5-FU/LV with or without oxaliplatin as adjuvant treatment following curative resection of gastric adenocarcinoma. Eur J Surg Oncol. 2011;37:466-72.

16. Catalano V, Bisonni R, Graziano F, Giordani P, Alessandroni $P$, Baldelli AM. A phase II study of modified FOLFOX as first-line chemotherapy for metastatic gastric cancer in elderly patients with associated diseases. Gastric Cancer. 2013;16:411-9.

17. Wang X, Wang Y, Qiu M, Li Q, Li ZP, He B, et al. Postoperative chemoradiotherapy in gastric cancer: a phase I study of radiotherapy with dose escalation of oxaliplatin, 5-fluorouracil, and leucovorin (FOLFOX regimen). Med Oncol. 2011;28(Suppl 1):S274-9.

18. Matzinger OGE, Bernstein Z, Maingon P, Haustermans K, Bosset JF, Gulyban A, et al. EORTC-ROG expert opinion: radiotherapy volume and treatment guidelines for neoadjuvant radiation of adenocarcinomas of the gastroesophageal junction and the stomach. Radiother Oncol. 2009;92:164-75.

19. National Cancer Institute. Common terminology criteria for adverse events v3.0 (CTCAE). http://ctep.cancergov/forms/ CTCAEv3.pdf. Accessed on 15 July 2005.

20. Caussanel JP, Lévi F, Brienza S, Misset JL, Itzhaki M, Adam R, et al. Phase I trial of 5-day continuous venous infusion of oxaliplatin at circadian rhythm modulate rate compared with constant rate. J Natl Cancer Inst. 1990;82:1046-50.

21. Kim TH, Park SR, Ryu KW, Kim YW, Bae JM, Lee JH, et al. Phase 3 trial of postoperative chemotherapy alone versus chemoradiation therapy in stage III-IV gastric cancer treated with R0 gastrectomy and D2 lymph node dissection. Int J Radiat Oncol Biol Phys. 2012;84:e585-92.

22. Zhu WG, Xua DF, Pu J, Zong CD, Li T, Tao GZ, et al. A randomized, controlled, multicenter study comparing intensitymodulated radiotherapy plus concurrent chemotherapy with chemotherapy alone in gastric cancer patients with D2 resection. Radiother Oncol. 2012;104:361-6.

23. Lee J, do Lim H, Kim S, Park SH, Park JO, Park YS, et al. Phase III trial comparing capecitabine plus cisplatin versus capecitabine plus cisplatin with concurrent capecitabine radiotherapy in completely resected gastric cancer with D2 lymph node dissection: the ARTIST trial. J Clin Oncol. 2012;30:268-73.

24. Wieland P, Dobler B, Mai S, Hermann B, Tiefenbacher U, Steil $\mathrm{V}$, et al. IMRT for postoperative treatment of gastric cancer: 
covering large target volumes in the upper abdomen: a comparison of a step-and-shoot and an arc therapy approach. Int J Radiat Oncol Biol Phys. 2004;59:1236-44.

25. Leong T, Willis D, Joon DL, Condron S, Hui A, Ngan SY. 3D conformal radiotherapy for gastric cancer-results of a comparative planning study. Radiother Oncol. 2005;74:301-6.

26. Soyfer V, Corn BW, Melamud A, Alani S, Tempelhof H, Agai R, et al. Three-dimensional non-coplanar conformal radiotherapy yields better results than traditional beam arrangements for adjuvant treatment of gastric cancer. Int $\mathbf{J}$ Radiat Oncol Biol Phys. 2007;69:364-9.

27. El-Hossiny HA, Diab NA, El-Taher MM. A comparative dosimetric study of adjuvant 3D conformal radiotherapy for operable stomach cancer versus AP-PA conventional radiotherapy in NCICairo. J Egypt Natl Cancer Inst. 2009;21:197-202.

28. Minn AY, Hsu A, La T, Fisher GA, Ford JM, Norton JA, et al. Comparison of intensity-modulated radiotherapy and 3-dimensional conformal radiotherapy as adjuvant therapy for gastric cancer. Cancer. 2010;116:3943-52.

29. Quero L, Bouchbika Z, Kouto H, Baruch-Hennequin V, Gornet JM, Munoz N, et al. Postoperative chemotherapy followed by conformal concomitant chemoradiotherapy in high-risk gastric cancer. Int J Radiat Oncol Biol Phys. 2012;83:574-80.

30. Leong T, Joon DL, Willis D, Jayamoham J, Spry N, Harvey J, et al. Adjuvant chemoradiation for gastric cancer using epirubicin, cisplatin, and 5-fluorouracil before and after three-dimensional conformal radiotherapy with concurrent infusional 5-fluorouracil: a multicenter study of the Trans-Tasman Radiation Oncology Group. Int J Radiat Oncol Biol Phys. 2011;79:690-5.

31. Boda-Heggemann J, Weiss C, Schneider V, Hofheinz RD, Haneder S, Michaely $\mathrm{H}$, et al. Adjuvant IMRT/XELOX radiochemotherapy improves long-term overall- and disease-free survival in advanced gastric cancer. Strahlenther Onkol. 2013;189:417-23.

32. Hofheinz RD, Wenz F, Lukan N, Mai S, Kripp M, Staiger W, et al. Oxaliplatin and capecitabine-based chemoradiotherapy for gastric cancer-an extended phase I MARGIT and AIO trial. Int J Radiat Oncol Biol Phys. 2009;73:142-7.

33. Orditura M, De Vita F, Muto P, Vitiello F, Murino P, Lieto E, et al. Adjuvant chemoradiotherapy in patients with stage III or IV radically resected gastric cancer: a pilot study. Arch Surg. 2010;145:233-8.

34. Osti MF, Agolli L, Bracci S, Monaco F, Tubin S, Minniti G, et al. Adjuvant chemoradiation with 5-fluorouracil or capecitabine in patients with gastric cancer after D2 nodal dissection. Anticancer Res. 2012;32:1397-402.

35. Chang JS, Lim JS, Noh SH, Hyung WJ, An JY, Lee YC, et al. Patterns of regional recurrence after curative D2 resection for stage III (N3) gastric cancer: implications for postoperative radiotherapy. Radiother Oncol. 2012;104:367-73.

36. Kilic L, Ordu C, Ekenel M, Yildiz I, Keskin S, Sen F, et al. Comparison of two different adjuvant treatment modalities for pN3 gastric cancer patients after D2 lymph node dissection: can we avoid radiotherapy in a subgroup of patients? Med Oncol. 2013;30:660. 